\title{
Crescimento, floração e frutificação de oliveiras jovens em altitude intermediária do Oeste de Santa Catarina, Brasil
}

\author{
Eduardo Cesar Brugnara ${ }^{1}$
}

\begin{abstract}
Resumo - Este trabalho teve como objetivo comparar o crescimento, a floração e a frutificação de oliveiras jovens dos cultivares Arbequina, Arbosana, Frantoio e Koroneiki plantadas em quatro localidades do oeste catarinense, em altitudes de aproximadamente $600 \mathrm{~m}$. Quatro experimentos foram instalados em 2013 e 2014 em Maravilha, São José do Cedro, Bom Jesus do Oeste e Guaraciaba. A altura e diâmetro das copas, floração e rendimento de frutos foram avaliados até o quinto ano. Todos os cultivares apresentaram bom crescimento, especialmente a 'Frantoio', comparados com as observações nos países tradicionais produtores de azeitonas. Nenhum cultivar floresceu em 2015 e pouca floração ocorreu em 2017, mas em 2016 houve floração em três locais. 'Frantoio' não floresceu. 'Arbequina' e 'Arbosana' foram os mais produtivos em 2016/2017. Os resultados sugerem que a adaptação à região de 'Arbequina', 'Arbosana' e 'Koroneiki' é parcial e a da 'Frantoio' é baixa.
\end{abstract}

Termos para indexação: Olea europaea; adaptação; precocidade.

\section{Growth, flowering and fruiting of young olive trees in mid-altitude in the west of Santa Catarina, Brazil}

Abstract - This study aimed to compare the growth, flowering and fruiting of young 'Arbequina', 'Arbosana', 'Frantoio' and 'Koroneiki' olives planted in four places of the west region of the state of Santa Catarina, situated at $600 \mathrm{~m}$ above sea level. Four experiments were installed in 2013 and 2014 in Maravilha, São José do Cedro, Bom Jesus do Oeste and Guaraciaba. The height and diameter of the canopies, flowering and yield were assessed until the 5th growth season. All cultivars grew well when compared to assessments of traditional olive producer countries, especially 'Frantoio'. All plants failed to flower in 2015 and few flowers were observed in 2017, but in 2016 there was blooming in three sites. 'Frantoio' did not bloom. 'Arbequina' and 'Arbosana' were the most productive in 2016/2017. The results suggest that the adaptation to the region is partial for 'Arbequina', 'Arbosana' and 'Koroneiki' and poor for 'Frantoio'.

Index terms: Olea europaea; adaptation; precocity.

A oliveira (Olea europaea L.) tem como origem provável o leste da região mediterrânea. Apesar de a espécie ter sido introduzida no Brasil na época da colonização, os maiores avanços com relação à produtividade foram alcançados nas últimas duas décadas, especialmente nos estados de Minas Gerais e Rio Grande do Sul. A espécie se desenvolve melhor em solos de textura moderadamente fina, bem drenados e aerados, com $\mathrm{pH}$ igual ou superior a 6,0 , além de exigir temperaturas baixas para que a diferenciação floral e o florescimento ocorram em abundância (NAVARRO GARCÍA et al., 2012; MESQUITA et al., 2012).

Em Santa Catarina, estudos iniciados no final da década de 1990 resultaram em dados promissores sobre o desempenho dos cultivares 'Arbequina', 'Arbosana' e 'Koroneiki' (DA CROCE et al., 2006). Para dar continuidade na qualificação deste conhecimento, este trabalho objetivou comparar oliveiras jovens de quatro cultivares no Oeste de Santa Catarina, em áreas de altitude intermediária (cerca de $600 \mathrm{~m}$ ), quanto a crescimento, florescimento e produção de frutos.

Foram instalados experimentos em quatro locais do estado de Santa Catarina: Local 1: Bom Jesus do Oeste (associação Neossolo eutrófico e Cambissolo eutrófico, altitude de 680m); Local 2: São José do Cedro (Cambissolo Háplico eutrófico, altitude de 590m); Local 3: Guaraciaba (Cambissolo Háplico eutrófico, altitude de 673m); e Local 4: MaraviIha (altitude de $650 \mathrm{~m}$ ). Os tratamentos foram os cultivares 'Arbequina', 'Arbosana', 'Koroneiki e 'Frantoio, distribuídos em delineamento em blocos ao acaso com cinco repetições de cinco plantas, exceto no local 4 onde foram utilizadas quatro repetições.

A correção do solo foi feita com 6t ha- ${ }^{-1}$ de calcário dolomítico classe $\mathrm{C}$, previamente ao plantio, seguida por escarificação. As oliveiras foram plantadas em novembro de 2013, com espaçamento de $7 \times 5 \mathrm{~m}$ nos locais 1 a 3 , e em agosto de 2014 com espaçamento de $7 \times 4 \mathrm{~m}$ no local 4 . Em janeiro de 2014 foi realizada uma aplicação de $130 \mathrm{~g}$ de fertilizante NPK (9-33-12) por planta. Em agosto de 2014 e em 2015 (janeiro e setembro) foram fornecidas $150 \mathrm{~g}$ do mesmo fertilizante. Depois disso foi realizada adubação com 45 e 30g planta- ${ }^{-1}$ de N (MESQUITA et al., 2012), na forma de ureia em novembro de 2015 e fevereiro de 2016, respectivamente. Nos ciclos seguintes a adubação N-P-K foi realizada com base em Mesquita et al. (2012) e em análises foliares. Em 2016, 
nos locais 2 e 3 foi reaplicado calcário em dose necessária para atingir $\mathrm{pH}$ 6,5. Entre o início do crescimento dos racemos florais e a abertura das flores foram realizadas pulverizações de $B$ e Ca. As plantas foram podadas regularmente para formação em vaso e pulverizadas para controle da lagarta Palpita forficifera, de cochonilhas e de manchas foliares. Além disso foram realizadas roçadas nas entrelinhas e controle de plantas espontâneas com enxada e herbicida nas coroas e, mais tarde, na linha de plantas (faixa).

Em fevereiro de 2016 e julho de 2018 foram realizadas avaliações da altura e do diâmetro médio das copas, cujos dados foram analisados por análises de variância e testes de Tukey $(\alpha=5 \%)$. A intensidade da floração foi avaliada por meio de uma escala com quatro escores: ausente (sem racemos florais), escassa (poucas plantas com racemos e, quando presentes, em poucos ramos), média (maioria das plantas com flores, mas poucos ramos floridos) e intensa (maioria das plantas e ramos com flores). Quando ocorreu a produção de frutos, a massa foi mensurada e os cultivares comparados pelo teste de Dunn após a análise de Kruskal-Wallis ( $\alpha=5 \%$ ). Todas as análises foram feitas separadamente por local e ano, utilizando-se o pacote estatístico $R$.

A altura das plantas foi afetada significativamente pelos tratamentos nos locais 1, 3 e 4, onde as plantas de 'Frantoio' estiveram entre as mais altas (Tabela 1). Porém, a significância das diferenças entre um dado par de cultivares foi dependente do local. No local 1 , por exemplo, em 2018 'Frantoio' tinha plantas mais altas que 'Arbequina' e 'Koroneiki', mas não diferia delas no local 3. O contrário aconteceu com a 'Arbosana'. Os genótipos também afetaram significativamente o diâmetro da copa nos locais 2, 3 e 4, o qual variou de 2,34 a 3,5m (Tabela 1). No local 1 as diferenças não foram significativas. Nos outros, 'Frantoio' sempre esteve entre as plantas com maior tamanho, e a 'Arbosana' entre as menores.

Os dados observados sugerem que o crescimento da oliveira nas condições edafoclimáticas em questão é satisfatório, comparado ao que se observa em países tradicionalmente olivicultores. Por exemplo, em Israel, sob irrigação, o cultivar Koroneiki apresentou cerca de $3,9 \mathrm{~m}$ de altura, respectivamente, aos cinco anos de idade (SCHNEIDER et al., 2012). A 'Arbequina', na Espanha, aos três anos, teve média de $2,8 \mathrm{~m}$ de diâmetro e $3 \mathrm{~m}$ de altura, passando para 3,2 e 3,6m aos quatro anos (VILLALOBOS et al., 2006). O crescimento naquelas condições pode ser considerado similar ao observado em Santa Catarina, pois pequenas diferenças podem ser devidas a podas que reduzem as dimensões da copa. O crescimento observado em Santa Catarina se deve às condições satisfatórias de fertilidade dos solos, após a correção, grande disponibilidade de água e temperaturas elevadas.

Na safra 2016/2017 houve floração e frutificação nos locais 1, 3 e 4 pelos cultivares Arbequina, Arbosana e Koroneiki (Tabela 2), que diferiram em intensidade, enquanto no local 2 não houve floração em nenhuma safra. Nos locais 3 e 4 a floração mais intensa ocorreu na 'Arbequina'. Nos mesmos locais 3 e 4 a Arbequina e a Arbosana produziram significativamente mais frutos que a 'Koroneiki' e a 'Frantoio', que não diferiram entre si em nenhum dos locais. Nas safras 2015/2016 e 2017/2018 não houve frutificação em nenhum dos cultivares e locais avaliados, apesar da emissão de poucas flores (escassa) por 'Arbequina', 'Arbosana' e 'Koroneiki' nos locais 1, 3 e 4 na safra 2017/2018 (dados não apresentados). A produção resultante não foi medida por ser insignificante.

Sendo o ciclo 2016/2017 a quarta estação de crescimento das plantas levadas ao campo em 2013, esperava-se colher a primeira safra de 'Arbequina', 'Arbosana' e 'Koroneiki' ainda em 2016, terceira estação de crescimento, como observado previamente em Chapecó, local de altitude semelhante, quando as plantas produziram 2,6 a 2,8kg por planta (DA CROCE et al., 2016). Da mesma forma se esperava produção em 2017/2018, apesar de a oliveira ser uma planta com forte alternância de produção (LAVEE, 2007), já que a produção anterior foi pequena. Uma possível explicação para a improdutividade nas duas safras é a falta de frio no período de repouso. Nos invernos de 2015 e 2017 o acúmulo de unidades de frio (UF) (DE MELO-ABREU et al., 2004) em São Miguel do Oeste, estação meteorológica localizada em altitude de $665 \mathrm{~m}$, foi de 23 e $34 U^{1}$, enquanto a estimativa da necessidade da 'Arbequina' é 339UF (DE MELO-ABREU et al., 2004). Já em 2016 o acúmulo foi de 133UF. A falta de frio inibe ou diminui a intensidade do florescimento (MALIK \& BRADFORD, 2005), pois a diferenciação floral das gemas induzidas se dá no período de baixas temperaturas (NAVARRO GARCÍA et al., 2012; MESQUITA et al., 2012). O acúmulo de frio insuficiente também explica a ausência de produção no Local 2, em todos os anos, pois é o de menor altitude. Apesar disso, não se pode descartar o efeito de outros fatores correlacionados ao frio, já que o efeito das horas ou unidades de frio na floração da oliveira ainda é controverso na literatura. Não foram encontradas estimativas precisas da necessidade dos cultivares 'Arbosana' e 'Koroneiki'. Porém, 'Frantoio' necessita 671UF (DE MELO-ABREU et al., 2004) e apresentou baixa intensidade de florescimento no norte da Argentina (391 a 859m de altitude), que foi atribuído à falta de frio (AYBAR et al., 2015).

$\mathrm{Na}$ Espanha se obteve até o quinto ano (2001 a 2004) produções acumuladas equivalentes a 14,5-24,0 kg planta $^{-1}$ em um olival superintensivo (DE LA ROSA et al., 2007). Ainda, mesmo com 780 plantas por hectare, a 'Arbequina' produziu $8,56 \mathrm{~kg}$ planta $^{-1}$ no terceiro ano (LEÓN et al., 2007). As médias observadas por Da Croce et al. (2016) em Chapecó variaram de 3,2 a $5,76 \mathrm{~kg} \mathrm{planta}^{-1}$. Todas as produções acima são superiores às observadas neste trabalho.

Assim, pode-se inferir que a adaptação das oliveiras 'Arbequina', 'Arbosana' e 'Koroneiki' na região de altitudes intermediárias do Oeste de Santa Catarina é parcial, especialmente por causa de anos desfavoráveis quanto ao acúmulo de frio, e que a 'Frantoio' não se adapta. Essas informações sugerem um estudo das condições climáticas históricas para identificar riscos climáticos associados à olivicultura da região, avaliações de cultivares em áreas com maior acúmulo de frio e de

${ }^{1}$ Calculadas a partir de dados disponibilizados pelo Instituto Nacional de Meteorologia, do governo brasileiro. 
Tabela 1. Altura e diâmetro médio da copa de cultivares de oliveiras 'Arbequina', 'Arbosana', 'Frantoio' e 'Koroneiki' no Oeste de Santa Catarina em dois momentos de avaliação (fevereiro de 2016 e julho de 2018)

Table 1. Canopy height and mean diameter in trees of 'Arbequina', 'Arbosana', 'Frantoio' and 'Koroneiki' olive cultivars in the west of Santa Catarina in two evaluation moments

(February 2016 and July 2018)

\begin{tabular}{cccccc}
\hline Local $^{(1)}$ & Cultivar & \multicolumn{2}{c}{ Altura $(\mathrm{m})$} & \multicolumn{2}{c}{ Diâmetro médio $(\mathrm{m})$} \\
\cline { 3 - 6 } & & 2016 & 2018 & 2016 & 2018 \\
\hline \multirow{2}{*}{ Local 1 } & Arbequina & $2,52^{\mathrm{ns}}$ & $3,24 \mathrm{~b}^{(2)}$ & $1,77^{\mathrm{ns}}$ & $2,600^{\mathrm{ns}}$ \\
& Arbosana & 2,64 & $3,50 \mathrm{ab}$ & 1,84 & 2,34 \\
& Frantoio & 2,79 & $4,16 \mathrm{a}$ & 2,25 & 2,82 \\
& Koroneiki & 2,40 & $3,38 \mathrm{~b}$ & 1,66 & 2,50 \\
\hline \multirow{2}{*}{ Local 2 } & Arbequina & $2,87^{\mathrm{ns}}$ & $3,52^{\mathrm{ns}}$ & $2,00 \mathrm{~ns}$ & $3,20 \mathrm{ab}$ \\
& Arbosana & 2,65 & 3,63 & 1,82 & $3,01 \mathrm{~b}$ \\
& Frantoio & 2,68 & 3,50 & 2,23 & $3,29 \mathrm{ab}$ \\
& Koroneiki & 2,63 & 3,63 & 2,04 & $3,39 \mathrm{a}$ \\
\hline \multirow{2}{*}{ Local 3 } & Arbequina & $1,41 \mathrm{~b}$ & $3,39 \mathrm{ab}$ & $1,82 \mathrm{~b}$ & $2,91 \mathrm{ab}$ \\
& Arbosana & $1,50 \mathrm{~b}$ & $3,19 \mathrm{~b}$ & $1,89 \mathrm{~b}$ & $2,48 \mathrm{~b}$ \\
& Frantoio & $1,95 \mathrm{a}$ & $3,82 \mathrm{a}$ & $2,26 \mathrm{a}$ & $3,32 \mathrm{a}$ \\
& Koroneiki & $1,51 \mathrm{~b}$ & $3,44 \mathrm{ab}$ & $2,00 \mathrm{ab}$ & $2,68 \mathrm{ab}$ \\
\hline \multirow{2}{*}{ Local 4 } & Arbequina & $2,69 \mathrm{a}$ & $4,19{ }^{\mathrm{ns}}$ & $1,86 \mathrm{ab}$ & $3,50 \mathrm{a}$ \\
& Arbosana & $2,26 \mathrm{~b}$ & 3,75 & $1,54 \mathrm{~b}$ & $2,80 \mathrm{~b}$ \\
& Frantoio & $2,67 \mathrm{a}$ & 3,99 & $2,18 \mathrm{a}$ & $3,38 \mathrm{ab}$ \\
& Koroneiki & $2,14 \mathrm{~b}$ & 3,68 & $1,45 \mathrm{~b}$ & $3,15 \mathrm{ab}$ \\
\hline
\end{tabular}

(1) Local 1: Bom Jesus do Oeste; Local 2: São José do Cedro; Local 3: Guaraciaba; Local 4: Maravilha; ${ }^{\text {ns: }}$ Diferença não significativa; ${ }^{(2)}$ : Médias seguidas pela mesma letra, por local, nas colunas não diferem estatisticamente (Tukey, $\alpha=5 \%$ ).

Tabela 2. Intensidade de floração e massa de azeitonas colhidas dos cultivares de oliveira Arbequina, Arbosana, Frantoio, e Koroneiki cultivadas em diferentes locais de Santa Catarina na safra 2016/17

Table 2. Flowering intensity and mass of olives harvested from Arbequina, Arbosana, Frantoio, and Koroneiki olive cultivars grown in different locations of Santa Catarina in the 2016/17 harvest season

\begin{tabular}{|c|c|c|c|c|c|c|}
\hline \multirow[t]{2}{*}{ Cultivar } & \multicolumn{2}{|c|}{ Local $^{(1)} 1$} & \multicolumn{2}{|c|}{ Local 3} & \multicolumn{2}{|c|}{ Local 4} \\
\hline & Floração & Produção & Floração & Produção & Floração & Produção \\
\hline & & (g planta ${ }^{-1}$ ) & & (g planta ${ }^{-1}$ ) & & $\left(\right.$ g planta $^{-1}$ ) \\
\hline Arbequina & Média & 44,65 a & Intensa & 306,10 a & Intensa & 88,63 a \\
\hline Arbosana & Média & $10,16 a b$ & Média & $33,80 \mathrm{a}$ & Média & $70,31 \mathrm{a}$ \\
\hline Koroneiki & Escassa & $6,20 a b$ & Escassa & $0,21 b$ & Média & $12,94 \mathrm{~b}$ \\
\hline Frantoio & Ausente & $0,00 \mathrm{~b}$ & Ausente & $0,00 \mathrm{~b}$ & Ausente & $0,00 \mathrm{~b}$ \\
\hline C.V. $(\%)^{(4)}$ & & 417 & & 458 & & 220 \\
\hline
\end{tabular}

(1) Local 1: Bom Jesus do Oeste; Local 2: São José do Cedro; Local 3: Guaraciaba; Local 4: Maravilha;

(2): Na safra 2018 não houve produção em nenhum local. No Local 2 não ocorreu floração; ${ }^{(3)}$ : Médias seguidas pela mesma letra dentro de cada local não diferem estatisticamente (teste de Dunn, $\alpha=0,05$ ); ${ }^{(4)}$ : C.V. = Coeficiente de variação.

uso de reguladores de crescimento para melhorar a floração e frutificação em casos de frio insuficiente.

\section{Agradecimentos}

Ao Programa SC Rural, à Fundação de Amparo à Pesquisa e Inovação do Estado de Santa Catarina, ao engenheiro florestal Dorli Mario da Croce e aos agricultores que foram parceiros nesta pesquisa e do rendimento de azeite das oliveiras 'Arbequina', 'Arbosana' e 'Koroneiki' em Santa Catarina. Agropecuária Catarinense, Florianópolis, v. 29, n. 1, p. 54-57, 2016.

DE LA ROSA, R.; LEÓN, L.; GUERRERO, N.; RALLO, L.; BARRANCO, D. Olival superintensivo - realidade ou utopia? Revista de Ciências Agrárias, Lisboa, v. 30, n. 1, p. 546551, 2007. Disponível em: https://goo.gl/ PNwzrb. Acesso em: 1 out. 2018.

DE MELO-ABREU, J. P.; BARRANCO, D.; CORDEIRO, A. M.; TOUS, J.; ROGADO, B. M.; VILLALOBOS, F. J. Modelling olive flowering date using chilling for dormancy release and thermal time. Agricultural and Forest Meteorology, Amsterdam, v. 125, n. 1-2, p. 121-127, 2004.

LAVEE, S. Bienial bearing in olive (Olea europaea). Annales: Series Historia Naturalis, Primorsko, v. 27, n. 1, p. 101-112, 2007. Disponível em: <https://bit.ly/2Is9Gix>. Acesso em: 12 mar. 2018.

LEÓN, L.; DE LA ROSA, R.; RALLO, L.; GUERRERO, N.; BARRANCO, D. Influence of spacing on the initial production of hedgerow 'Arbequina' olive orchards. Spanish Journal of Agricultural Research, Madrid, v. 5, n. 4, p. 554-558, 2007. Disponível em: <https:// bit.ly/2NVVhR1>. Acesso em: 30 jul. 2018.

MALIK, S. A. N.; BRADFORD, J. M. Is chilling a prerequisite for flowering and fruiting in 'arbequina' olives? International Journal of Fruit Science, Filadélfia, v. 5, n. 3, p. 29-39, 2005.

MESQUITA, H. A.; NAVARRO GARCÍA, C.; CISTA, E. L. Solos, aspectos nutricionais e sugestões de fertilização. In: OLIVEIRA, A. F. (Ed.). Oliveira no Brasil: tecnologia de produção. Belo Horizonte: Epamig, 2012. p. 385-433.

NAVARRO GARCÍA, C.; MESQUITA, H. A.; ALVARENGA, A. A. Limitações de clima, solo e planejamento do plantio para o cultivo da oliveira. In: OLIVEIRA, A. F. (Ed.). Oliveira no Brasil: tecnologias de produção. Belo Horizonte: Epamig, 2012. p. 349-383.

SCHNEIDER, D.; GOLDWAYA, M.; BIRGERB, R.; STERNA, R. A. Does alteration of 'Koroneiki' olive tree architecture by uniconazole affect productivity? Scientia Horticulturae, Amsterdam, v. 139, p. 79-85, 2012. Disponível em: <https://bit.ly/2xPL2V4>. Acesso em: 30 jul. 2018.

VILLALOBOS, F. J.; TESTI, L.; HIDALGO, J.; PASTOR, M.; ORGAZ, F. Modelling potential growth and yield of olive (Olea europaea L.) canopies. European Journal of Agronomy, Conthey, v. 24, p. 296-303, 2006. Disponível em: <https://bit.ly/2xnWuqF>. Acesso em: 31 jul. 2018. 